\title{
Identification of Mosaic Order Area of Geometric Triangular Chiral Hexagonal Complexes in Interphase with Morphology Biosignature Pattern in the Interior of Martian Nested Impact Craters
}

\author{
Jairo A. Diaz ${ }^{1,2,3}$, Karen E. Paris ${ }^{2,3}$ \\ ${ }^{1}$ Laboratory of Pathology, Hospital Departmental of Villavicencio, Villavicencio, Colombia \\ ${ }^{2}$ Hospital Departmental of Granada, Meta, Colombia \\ ${ }^{3}$ Department of Pathology, School of Medicine, University Cooperative of Colombia, Villavicencio, Colombia \\ Email: jaditod@hotmail.com
}

Received 4 February 2014; revised 2 March 2014; accepted 10 March 2014

Copyright (C) 2014 by authors and Scientific Research Publishing Inc.

This work is licensed under the Creative Commons Attribution International License (CC BY). http://creativecommons.org/licenses/by/4.0/

(c) (i) Open Access

\section{Abstract}

From evolutionary miniaturization activation of ancestral larger genes stocks, an electromagnetic field derivate from cancer microscopic collision events participates in the elaboration of geometric complexes and chiral biomolecules that serve to build bodies with embryoid print as it develops during gestation. This miniaturization platform literally allows us to see what would otherwise remain completely invisible. In concordance with our observations collision extreme chaos generates in space time interval geometric scalable invariant extreme order. To determine whether our predictions are valid, we select Mars one of the planets with highest rate collision impact craters. The idea that impact events produce major geological effects that go far beyond the production of craters has recently been emphasized. We wonder if we could predict geometric chiral triangular hexagonal complexes in Martian landscape similar to those documented at microscopic, macroscopic, megascopic levels. We resolved to investigate the geomorphology patterns of more than 4000 collision impact craters in Mars landscape using images from Google Mars platforms, and HIRISE (High resolution imaging science experiment camera from the University of Arizona) based on a pattern recognition images algorithm we identified Mars mosaic order area (MOA). MOA is a circular cluster of overlap craters organized in apparent visible cycle sequential order oriented counterclockwise and consisting of nine craters that structure visible and measurable geometry in interface with biosignature morphologies in their interior not having been previously documented. Crater, therefore, is a step forward in understanding how collisions influence life, both on Earth and on other planets. We were pleased that the correct pattern image algorithm 
predicted the identification of irreducible geometry matrix of GTCHC complexes in Martian impact craters. Cancer can inform astrobiology.

\section{Keywords}

\section{Geometric Triangular Chiral Hexagon Complexes; Mars}

\section{Introduction}

Cancer is an excellent model to study chaotic systems and the most spectacular biological time machine of reverse information, that in an activated collective memory of ancestral genes, carried data linking the origin of cancer to the origin of life and the developmental processes of embryos. Recursive ontogeny recapitulates an earlier stage of development of multicellular organisms. Cancer recreates, in a step-by-step manner, the primitive self-assembly organization that emerges from collision extreme chaos entropy with predictable signature of irreducible geometry matrix and morphology biosignature pattern.

(1) Identification of Irreducible geometry matrix and morphology biosignature pattern at microscopic level. (mm):

We described and documented the self-assembly of geometric triangular chiral hexagon crystal-like complex organizations (GTCHC) in human pathologic tissues at macroscopic and microscopic levels, mainly in cancer processes [1]. We also documented and gathered insights into the magnetic field (MF) in cancer tissues and how it generates a functional geometric attractor complex in their entangled state [2], as well as a structural template platform on which intercellular cancer collisions generate an ejected crystal comet tail effect with fractal interface embryoid body reassembly transformation [3].

An electromagnetic field derivate from microscopic collision events participates in the elaboration of geometric complexes and chiral biomolecules that serve to build bodies with embryoid print as it develops during gestation [4] (Figures 1-4).

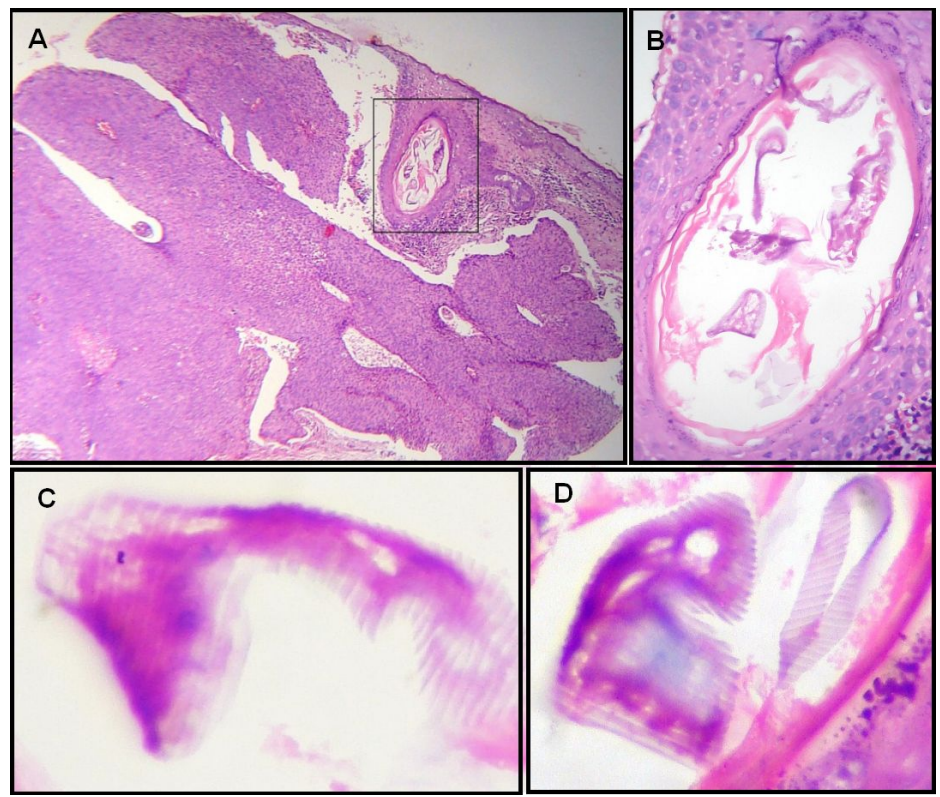

Figure 1. (A) Show panoramic view of malignant epithelial tumor. Observe the hole-crater tissue like pattern in the upper right area. (B) Hole tissue close up illustrate in their interior primordial DNA band like helices patterns emerging from the wall of the structure. (C) and (D) Visible DNA like helical bands that emerge from the wall of the tissue hole like craters are the responsible for structuring cancer embryo genic bodies. 


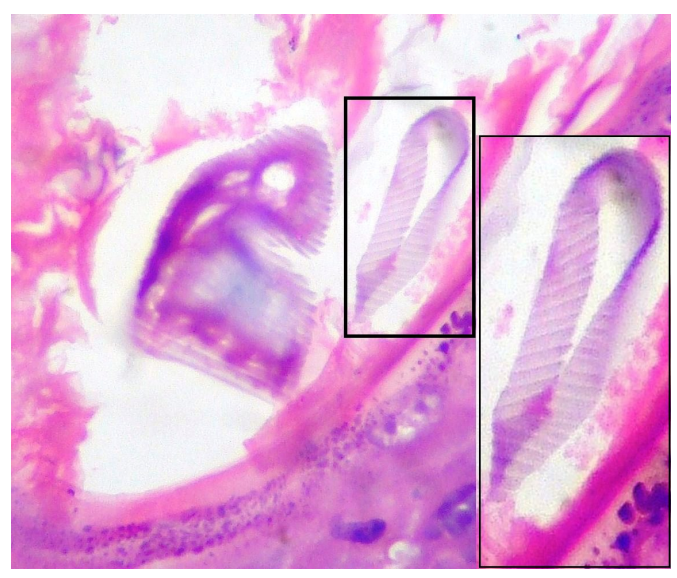

Figure 2. Illustrate Visible DNA helical like bands, structural platform of the embryoid bodies.

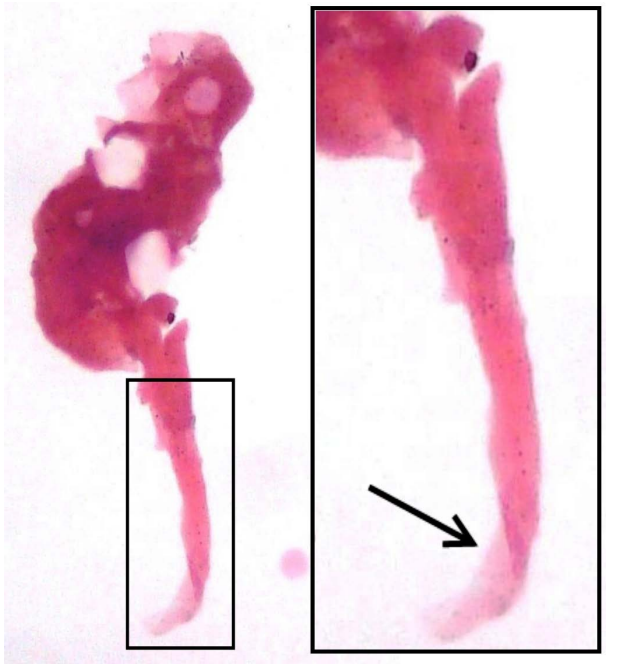

Figure 3. Illustrate two embryoid bodies assembled from a visible single chain of DNA helical like band.
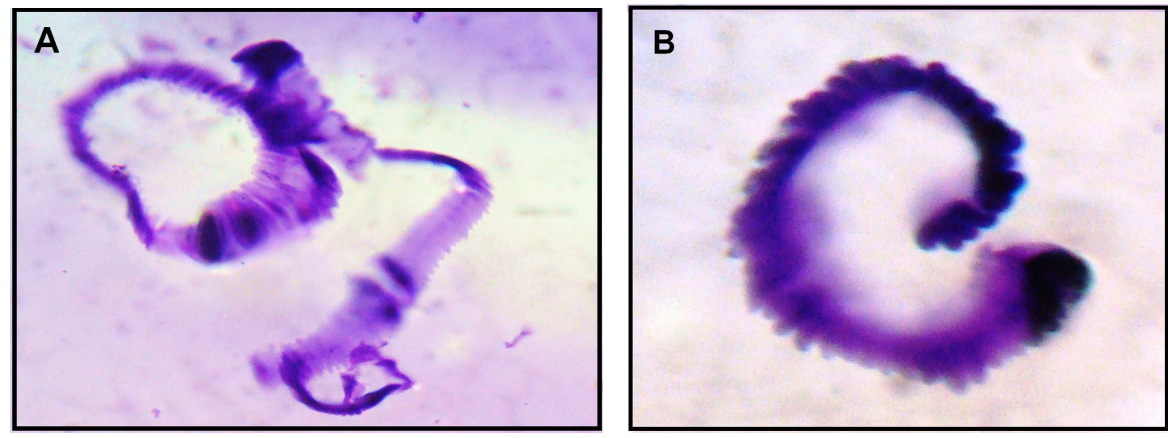

Figure 4. (A), (B) illustrate two embryoid bodies assembled from a visible single chain of DNA helical like band.

(2) Identification of Irreducible geometry matrix and morphology biosignature pattern at megascopic level. $(\mathrm{Km})$ :

The authors have described and documented fractal self-assembly of geometric triangular chiral hexagonal crystal-like complex organizations (GTCHC) and interface comet tail effect patterns in cancer processes. Ac- 
cording to this novel observation, cancer incorporates a real visualization world with a great surprising finding in biology, physics, and geology. This miniaturization platform literally allows us to see what would otherwise remain completely invisible. From theory to practice, this irreducible geometric matrix algorithm allowed us to identify in earth collision impact crater, geology, real measurable green infrared-electromagnetic stripe line in interface with hexagonal geomorphic pattern, triangular chiral pyramidal rock structures, well-defined geological mirror images, and template platforms that structure collision bodies characterizing ancestral primitive embryoid-like shape pattern embedded as giant fossils in rocks that have never been seen before (Figures 5-10).
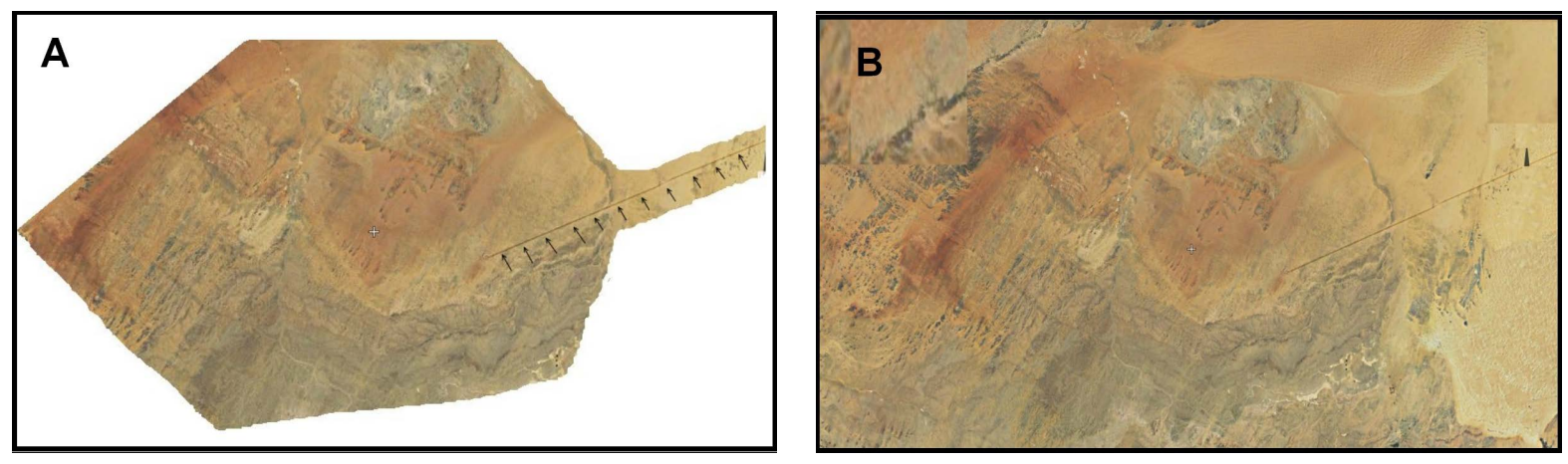

Figure 5. Altitude 30 miles. (A) is a detachment of image; (B) that show satellite sequential fractal external eco vibrational hexagonal geology pattern in interface with electromagnetic field stripe line generated by earth collision impact event.
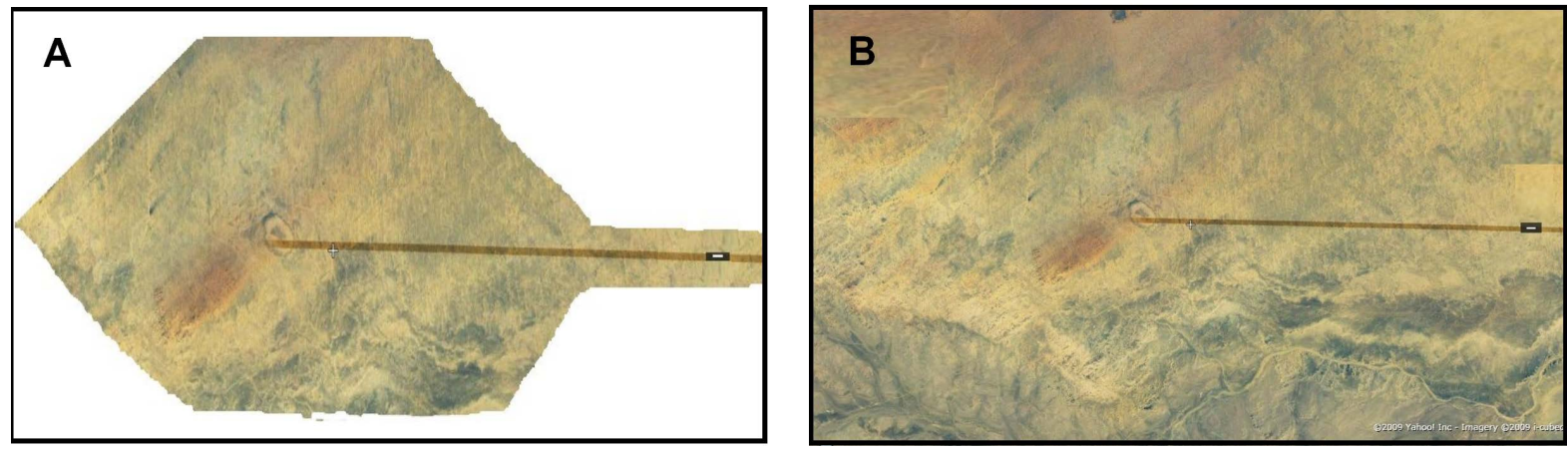

Figure 6. Altitude 15 miles. (A) is a detachment of image; (B) that show satellite sequential fractal medial eco vibrational hexagonal geology pattern in interface with electromagnetic field stripe line generated by earth collision impact event.

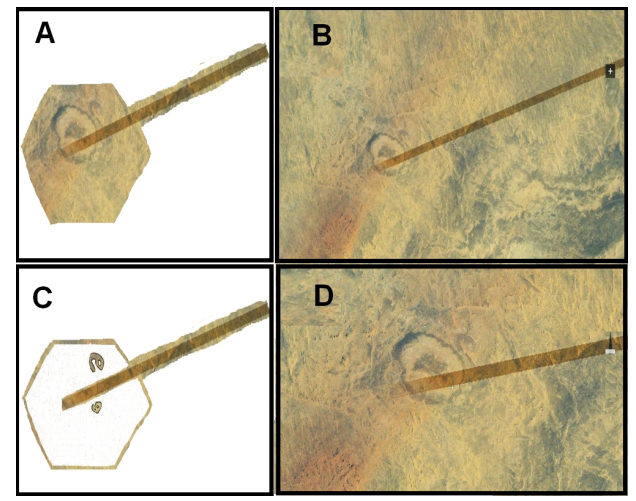

Figure 7. Altitude 6 miles (A) is a detachment of image; (B) that show satellite sequential fractal internal eco vibrational hexagonal geology pattern in interface with electromagnetic field stripe line generated by earth collision impact event. Altitude 3 miles (C) is a detachment of image (D) that show satellite sequential fractal core eco vibrational hexagonal geology pattern in interface with electromagnetic field stripe line generated by earth collision impact event. 


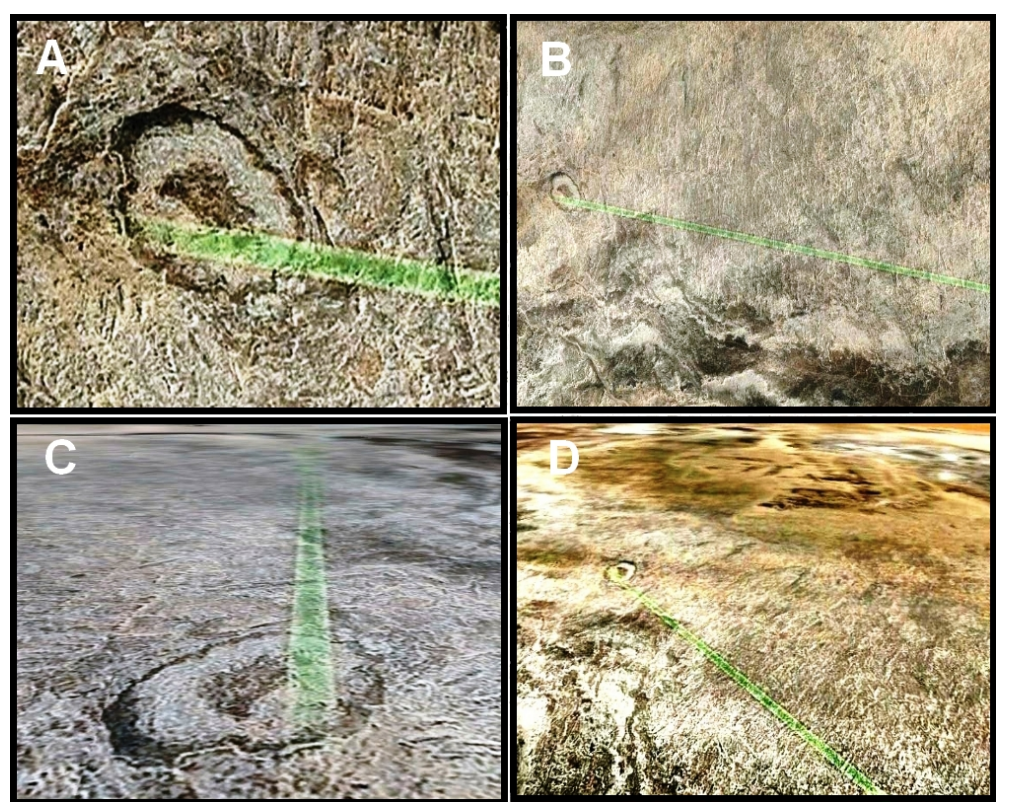

Figure 8. (A) Show identification of novel megascopic collision event, Satellite image show close up of green stripe line $128 \mathrm{~km}$ ending in convergence with hexagonal geomorphic and circular structure bull's-eye. (B), (C), (D) Satellite image show the geospatial effect interaction of the green stripe line over the surface terrain in terms of generation of helicoidally pattern throughout the trajectory of the line.
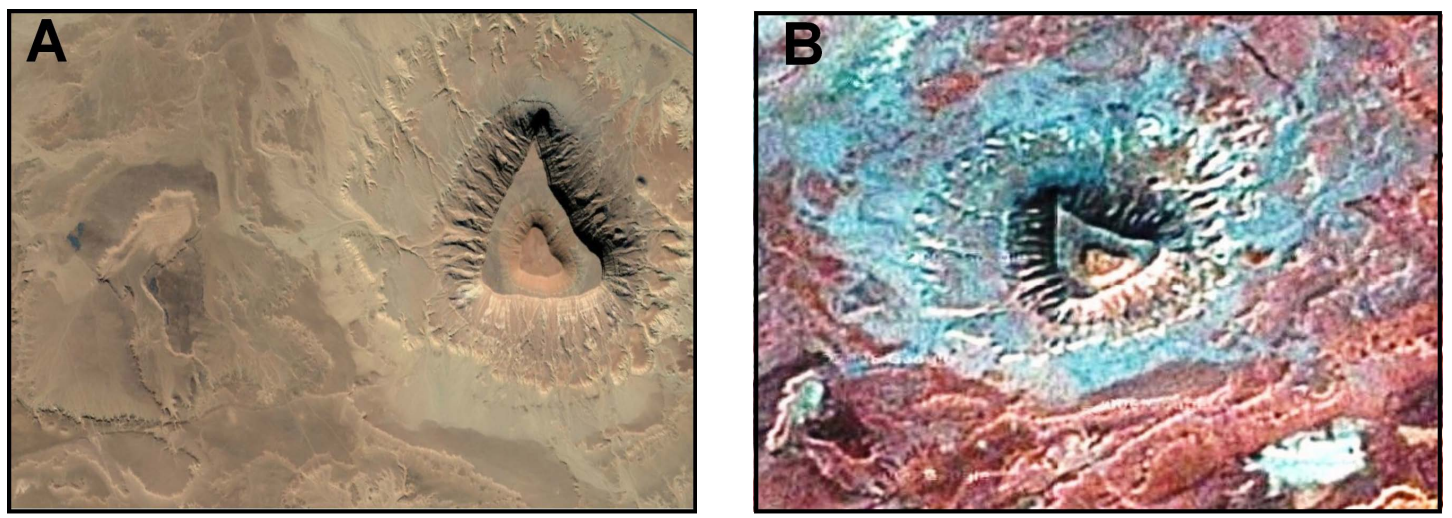

Figure 9. (A) Satellite image show pyramidal triangular self-assembly geologic structure with their chiral counterpart adjacent side by side. (B) Satellite image show close up of pyramidal triangular self-assembly geologic structure with a height of 550 meters and a width of 645 meters.

The electromagnetic field that is released in collision-impact events generates a fractal scalable invariant order of geometric triangular chiral hexagonal structures in the matrix interphase. The laws of biology and geology can finally be redirected to the laws of physics; specifically, magnetic fields create a new kind of classification based on these fractal structural similarities of the relationship geometric self-assembly geological structures, ancient sediments and rocks that could provide insights into antecedents of life [5].

(3) Predictable identification of Irreducible geometry matrix and morphological biosignature patterns on Mars collision impact craters. (140 million miles):

The same observational evidence is available to observers at different scalable locations. Isotropy means that the same observational evidence is available by looking in any direction in the universe as the same physical laws that apply throughout different places will appear similar to one another at all points in space and ought to experience the same physical development, which are correlated in time in such a way that all points at a certain distance from an observer appear to be at the same stage of development. In that sense, all spatial conditions in 

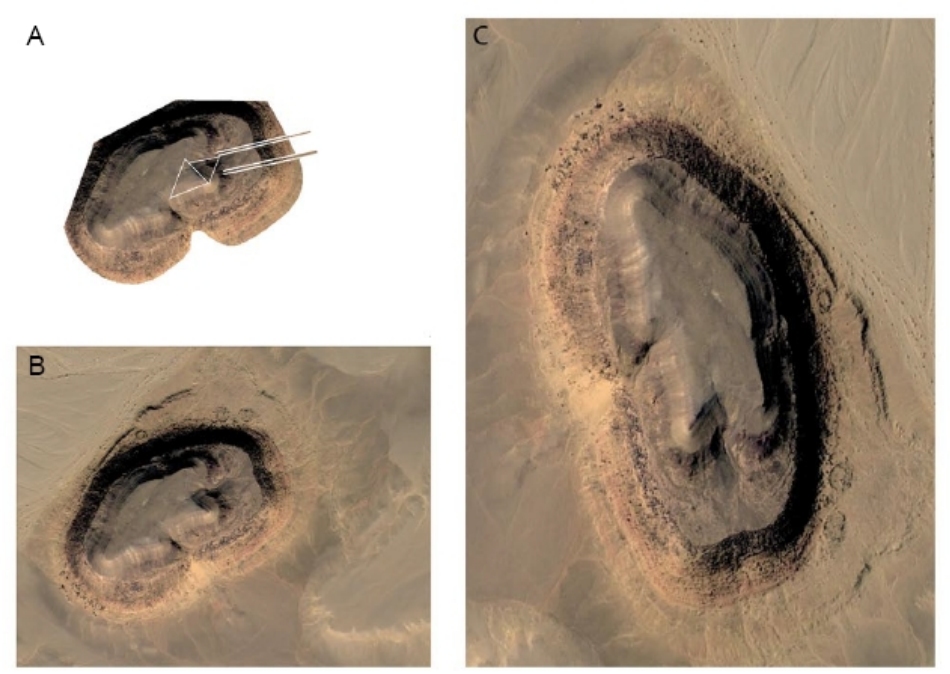

Figure 10. Aerial photography. (A) show detachment of (B), horizontal position of image (C), illustrating Rock collision body embryo morphism.

the Universe must appear to be homogeneous and isotropic to an observer at all times in the future and in the past earlier times, thus we propose in this article that GTCHC complexes have this property, which can be universal. In concordance with our observations, extreme chaos generates in space-time interval extreme order. To test this hypothesis, we selected Mars as one of the planets with the highest rate of impact craters, according to a University of Colorado, Boulder research team recently finished counting, outlining and cataloging a staggering 635,000 impact craters on Mars that are roughly a kilometer or more in diameter [6] Thus, Mars planet is the ideal scenario to study physics collisions, also morphological preservation of craters is cleaner and sharper on Mars that remain almost unchanged in the slow sedimentation, unlike what happens in Earth that the high erosion and sedimentation becomes difficult location, It's just that Earth's atmosphere, life forms and tectonic activity obliterate its craters or make them difficult to see. Mars should provide a window into our own planet's past.

Impact cratering produces not only crater form topographic features, but also structural disturbances at the site of impact, as well as a spectrum of transformed and newly formed rocks. The term "coptogenesis" (from the Greek wopto, to destroy by shock) may be used collectively to describe the impact process-a process fundamental to all cosmic bodies. Principal coptogenic topographic features of terrestrial impact craters may be subdivided into excavational, structural, and accumulative landforms, most of which subsequently experience various processes of degradation. Nevertheless, the original shape of craters may, in some cases, be reconstructed and compared with fresh craters on other planets. An immediate conformity between the pre-erosional topographic features of complex terrestrial craters and the morph structural elements of their erosional remnants is not a standing rule. Geological observations show that the inner structure of the proximal crater fill and distal ejecta is characterized by pseudo-stratification, and that these materials represent a group of facies of impactderived and impact-related, or coptogenic, lithologies [7]. The idea that impact events produce major geological effects that go far beyond the production of craters has recently been emphasized. We wonder if we could identify and document geometric chiral triangular hexagonal complexes in Martian landscape identical to what have been documented at microscopic, macroscopic, and megascopic levels.

\section{Materials and Methods}

Remote sensing is the acquisition of information about an object or phenomenon, without making physical contact with the object. In modern usage, the term generally refers to the use of aerial sensor technologies to detect and classify objects on earth, both on the surface and in the atmosphere and oceans. Satellite platforms, such as Google Earth, are valid scientific tools at the disposal of researchers in multiple disciplines, from ruins to the earth impact events [8]. Based on known pattern recognition image algorithm background and our 6-year experience of having methodologically and sequentially documented macroscopic and microscopic geometric patterns in malignant cancer tissues, and in the existence and documentation of real visible geologic GTCH C com- 
plexes previously documented, we resolved to investigate the geomorphology patterns of more than 4000 collision impact craters in the Mars landscape using images from Google Mars platforms, and HIRISE (High-Resolution Imaging Science Experiment camera from the University of Arizona). HiView is the best way to explore images of the Martian surface at the full resolution of the imagery generated by the Mars Reconnaissance Orbiter.

\section{Results}

Like a needle in a haystack, we identified, in that monotonous sea of craters in Mars, a mosaic order area (MOA).

MOA is a circular cluster of overlap craters organized in apparent visible cycle sequential order oriented counterclockwise and consisting of nine craters structuring visible and measurable geometry in interface with collision body morphologies in their interior, which have not been previously documented.

Documentary evidence:

The figure shows a panoramic view of MOA (zoom 1) consisting of an overlap of nine impact craters arranged in a semi-circle fashion (Figure 11, Figure 12).

Crater 1: Structuring in their interior a geometric triangular pattern with its base located on the top. Crater 2: Structuring in their interior a geometric triangular pattern with its base located in the bottom is evident of a chiral mirror image with the triangular structure located in Crater 1. Crater 3 structuring is a geometric hexagonal pattern that generates in their framework order stratification of helicoidal bands (Figure 13, Figure 14, Figure 15, Figure 16).

Craters 4, 5, and 6: From this hypertrophic helicoidal bands emerge collision body structures organized in a visible sequential growth of development (Figure 17).

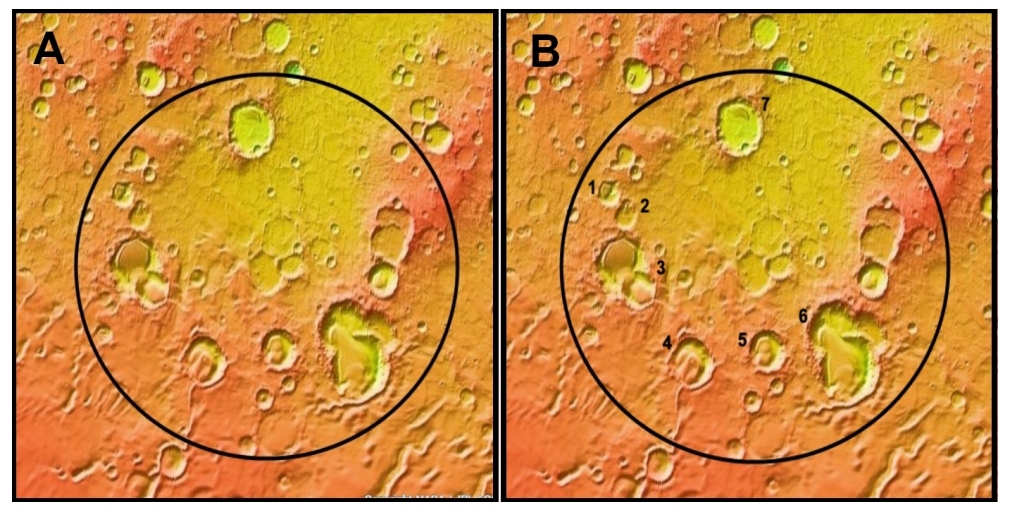

Figure 11. (A), (B) Mars HIRISE elevation image that illustrate MOA, Mars mosaic order area, a circular cluster of overlap craters organized in apparent visible cyclic sequential order oriented counterclockwise and consisting of nine craters that structuring visible and measurable geometry in interface with collision body biosignature pattern.

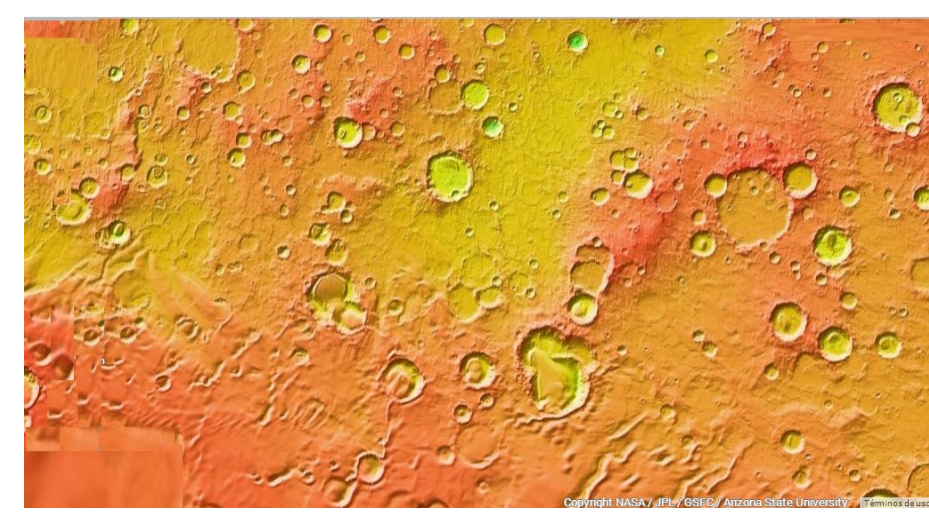

Figure 12. Mars HIRISE elevation images that illustrate MOA, Mars mosaic order areapanoramic view. 


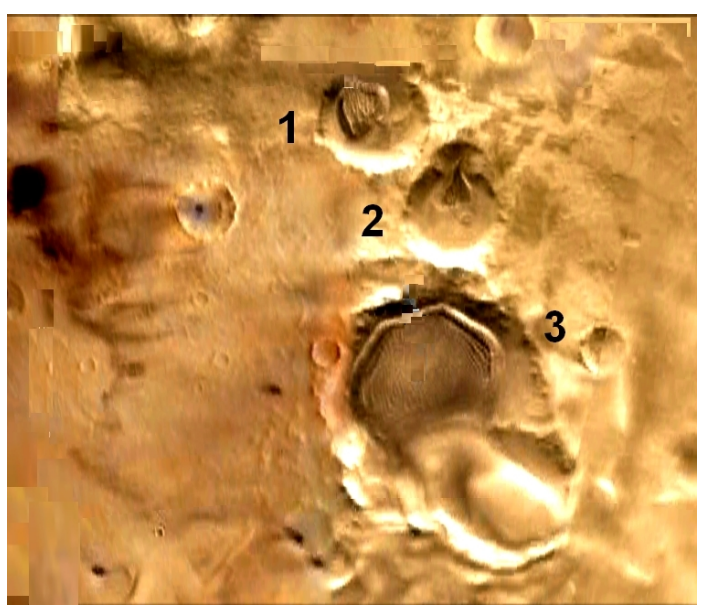

Figure 13. Mars HIRISE elevation image that illustrate MOA, 1, 2, 3 impact craters with geometric triangular chiral hexagon complexes.

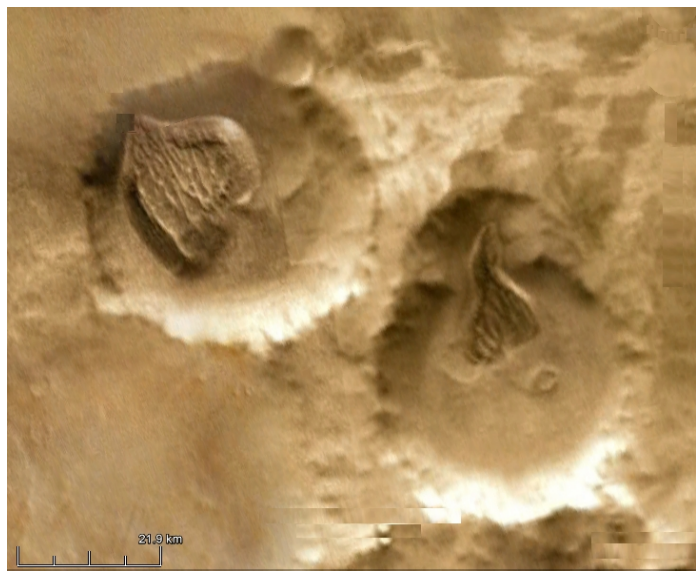

Figure 14. Mars HIRISE image that illustrate MOA impact craters with triangular chiral pattern.

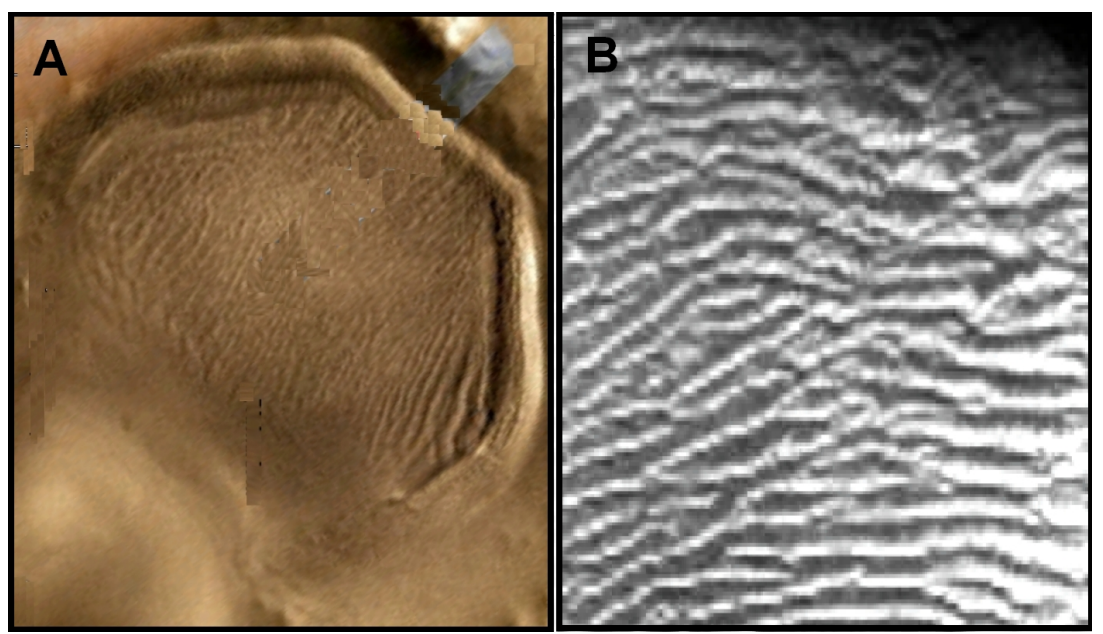

Figure 15. (A) (B) Mars HIRISE image that illustrate MOA impact crater with hexagonal pattern that generates in their framework wind order stratification of helicoidal bands. 


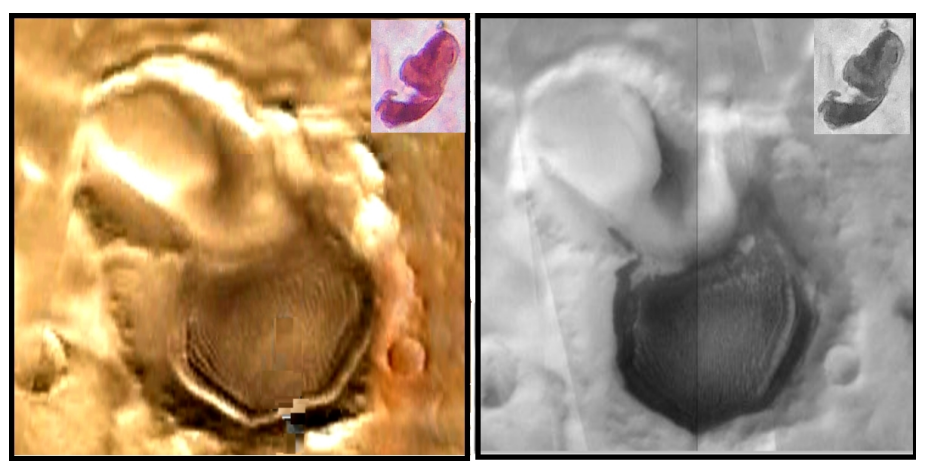

Figure 16. From windhypertrophic helicoidalbands emerge collision body biosignature pattern structure.

Crater 7: Structuring in their interior a well-developed collision body with dipole organization inside it makes possible the identification of helicoid bands (Figure 18). Crater 8: Adjacent to the semicircle is a well-differentiated collision body with an umbilical cord-like attachment to the wall of the crater that is reminiscent of an embryoid body (Figure 19).

Crater 9 shows the most well-elaborated growth collision body head tail-like embryoid structure and shows how their assemblage emerges from a hexagonal geometry platform (Figure 20, Figure 21). One can imagine the processing as the assembly line where geometry its computed first, the output is then send downstream that make a subsequent computation with a final dipole self-assembly product, result of memory association.

In our survey, the identification of other geometric patterns inside craters that were not previously documented was possible: Crater with a complex of three triangles inside, in which the great original triangular structure gives rise to two self-similar smaller fractal aligned scalable triangles, identical in disposition to the Giza pyramid complex (Figure 22, Figure 23, Figure 24, Figure 25). Another dynamic characteristic of Mars craters is the visible dipole energy generated in their interior. Due to an inward and upward motion of the target rocks, accompanied by compression and accretion, the uplift displays a system of intricate small folds, faults, and thrusts reflecting mostly the last phase of centripetal motion. Expansion or centrifugal energy in one semicircle crater area is balanced with contraction centripetal energy in the opposite one. Expansion-centrifugal zones show well-defined edge rim wall borders with star-like patterns of dissemination, whereas contraction-centripetal zones show evidence of stratification hyperdensities with flattened edges (Figure 26, Figure 27). From the wall of contraction areas emerge collision bodies formed by the hypertrophy of identical helicoid bands comparative with the embryoid bodies documented by us in cancer tissues, and derived from DNA-like helical bands (Figure 28, Figure 29, Figure 30).

\section{Discussion}

There's a story always hidden in crystals and rocks, and we describe three different phenomena occurring on scales of $1 \mathrm{~mm}, 100 \mathrm{~km}$, and almost a light year The similarity exists in the spatial domain; that is, not only are there obvious spatial similarities but the power spectra of the three phenomena are also nearly identical enough to find the tools that we need to interrogate those rocks and crystals to find what story they carry. As with any fingerprint, the pattern does not tell you much if you do not have anything to compare it to. Using our resources here, we find correlations and work allows us to really compare the GTCHC Mars samples with known patterns images in biologicalcancer chaos system.

We have documentary evidence to prove that GTCHC complexes are real structures derived from real events and not from computational models or mathematical physics formulas; [9] they are visible, measurable, reproducible, but most importantly, predictable in the residual detritus of collision impact events that these structures have the potential to preserve older historical states, and thus, to serve as a window into the human past.

Geometry and bio morphological patterns documented in this manuscript was captured from HIRIS-HighResolution Imaging Science Experiment camera University of Arizona-and generated by the Mars Reconnaissance Orbiter. The identification of these structures was performed with the help of pattern recognition images algorithm. We know the exact location coordinates of these structures, there are real visible, and measurable units. 


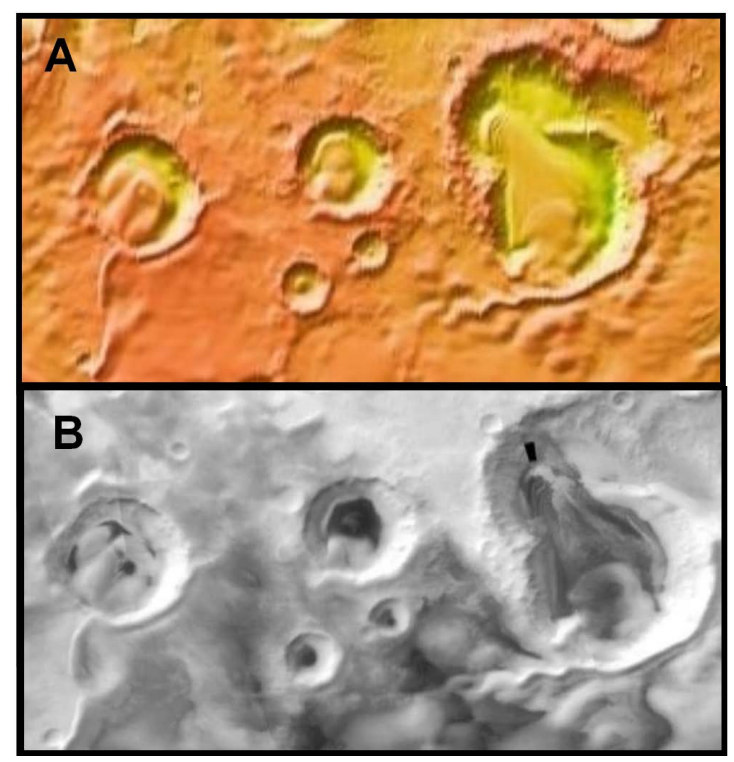

Figure 17. (A), (B) From hypertrophic helicoidal bandsemerge collision body biosignature pattern structures organizedin visible sequential growth of development.

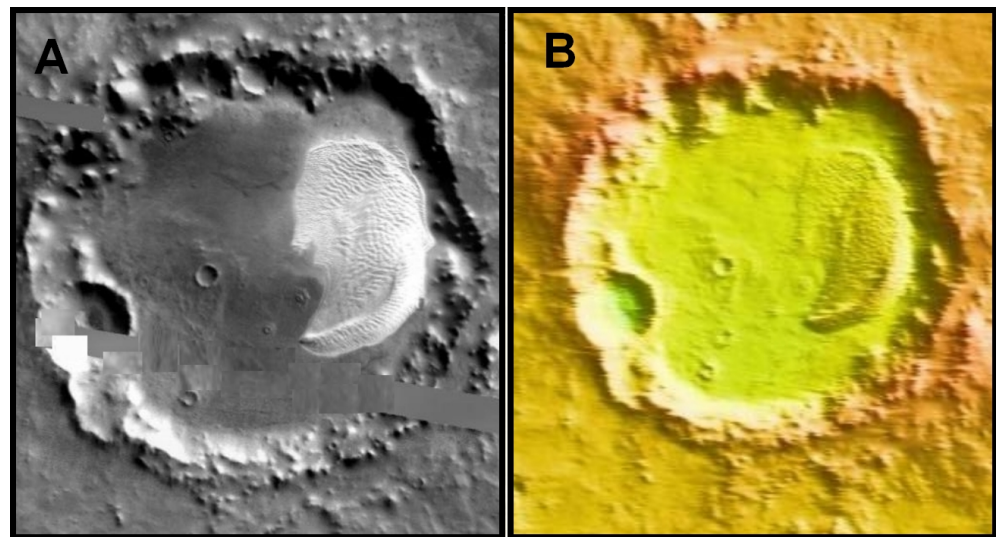

Figure 18. (A), (B) Illustrate crater 7, structuring in their interior well develop collision body biosignature pattern with dipole organization. Inside its visible the structuration of helicoids bands.

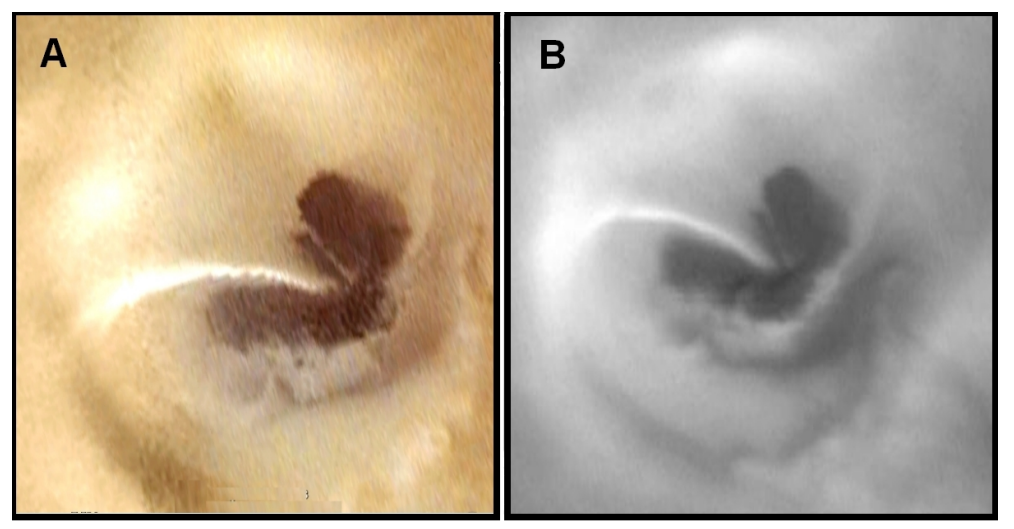

Figure 19. (A), (B) Illustrate crater 8, documenting one tied embryological structure attached to the wall by a like umbilical cord. 

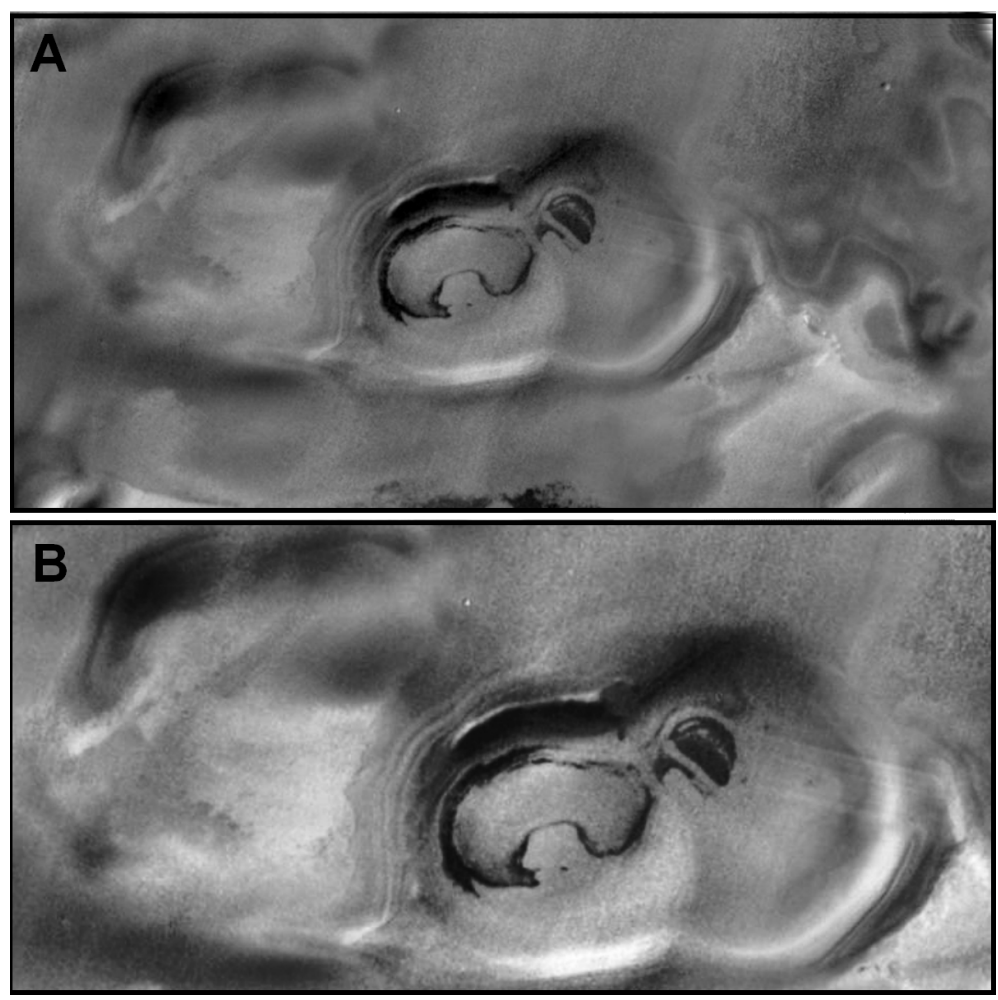

Figure 20. Mars HIRISE infrared image that illustrate crater 9 showing in the interior the most well elaborate growth collision body structure head tail biosignature pattern, documenting how their assembly emerge from hexagonal geometry platform.

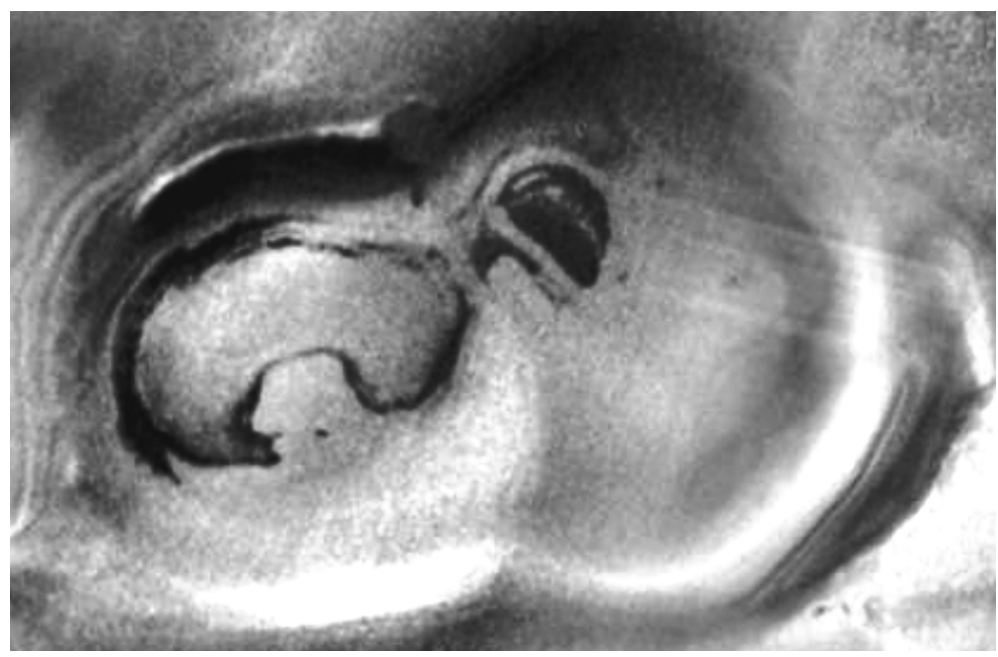

Figure 21. Mars HIRISE infrared image that illustrate crater 9 close up that show in the interior amazing and incredible head tail embryonic body structure never seen before. Its evident how is assembly this structure by the impact crater wall vibrations in response to light of different frequencies.

To assess the significance of a morphological finding, it must withstand the rigor of qualitative analysis of location, dimension, space, volume, as well as patterns of organizational dynamics and morphology integration of the structure examined with the surrounding environment; discern factors that allow an optical illusion and a real measurable structure. 


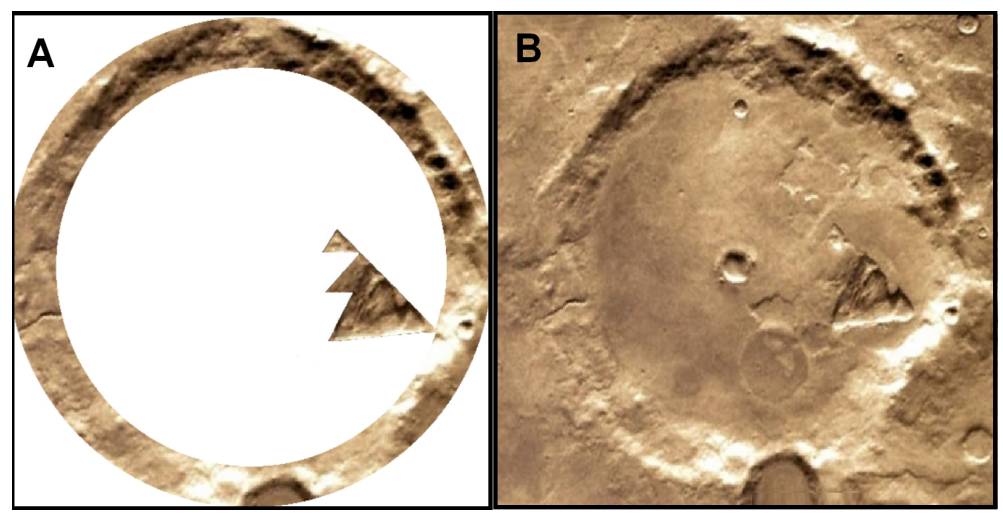

Figure 22. Crater with a complex of three triangles inside, from the great original triangle structure gives rise to two self-similar smaller fractal aligned scalable triangles identical in disposition with Giza pyramids complex not previously documented.

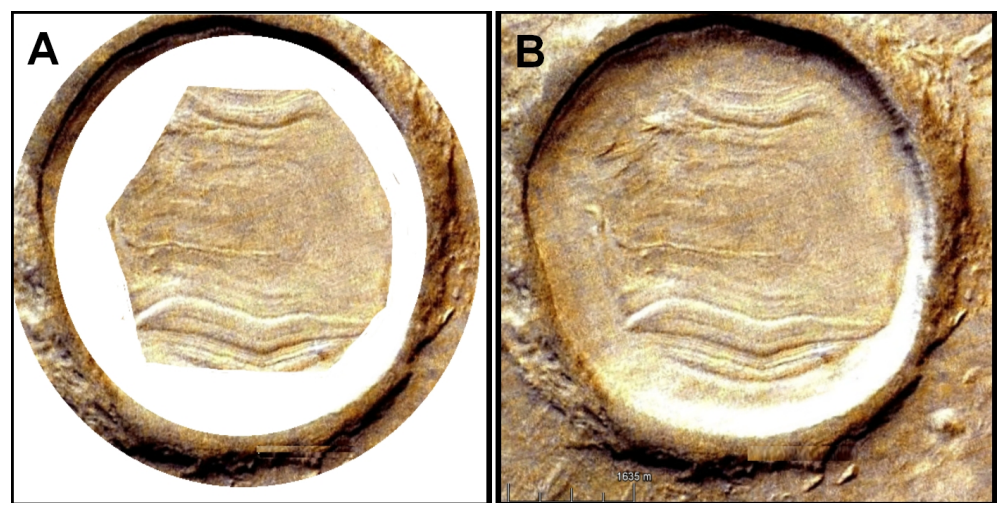

Figure 23. (A), (B) Crater with hexagonal pattern in their interior.

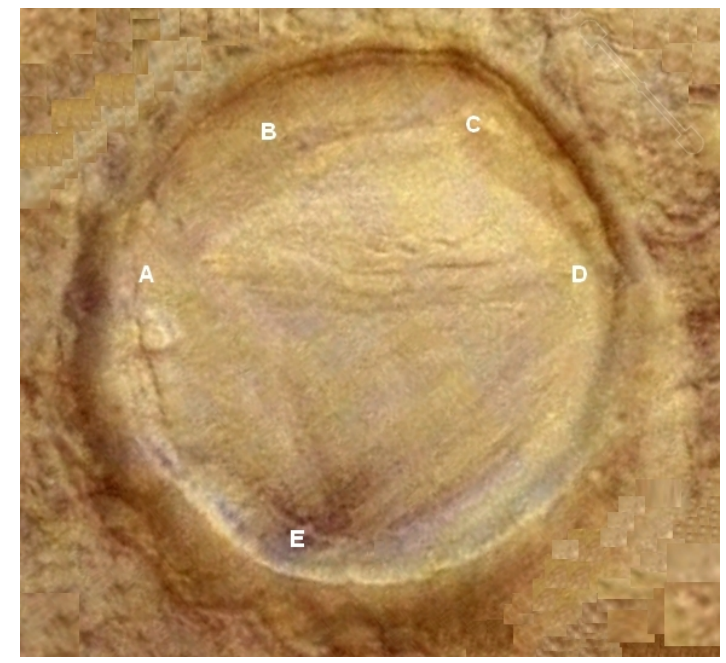

Figure 24. Crater with rhomboid pattern in their interior.

Head tail-like embryoid body structure analysis:

a) Embryoid body structure it's nested in the interior of pair-twin collision craters (Figure 20, Figure 21).

b) Collision craters produces in their interior, contraction dark and expansion white areas, clearly recognizable. 

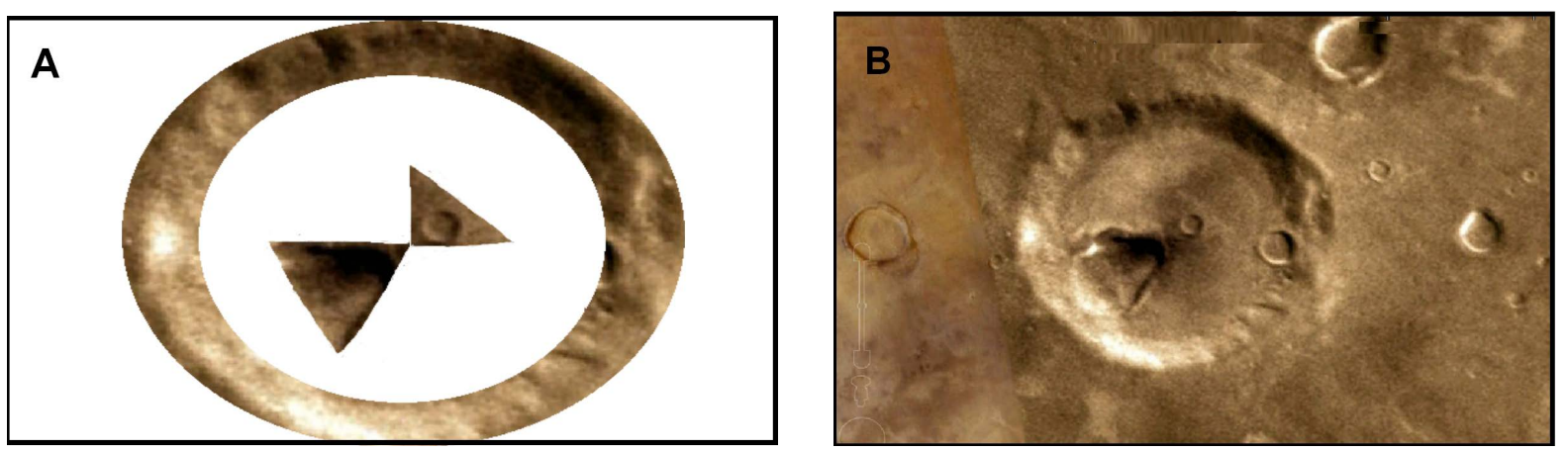

Figure 25. A it's a detachment of B that show Crater with triangular chiral mirror image in their interior.
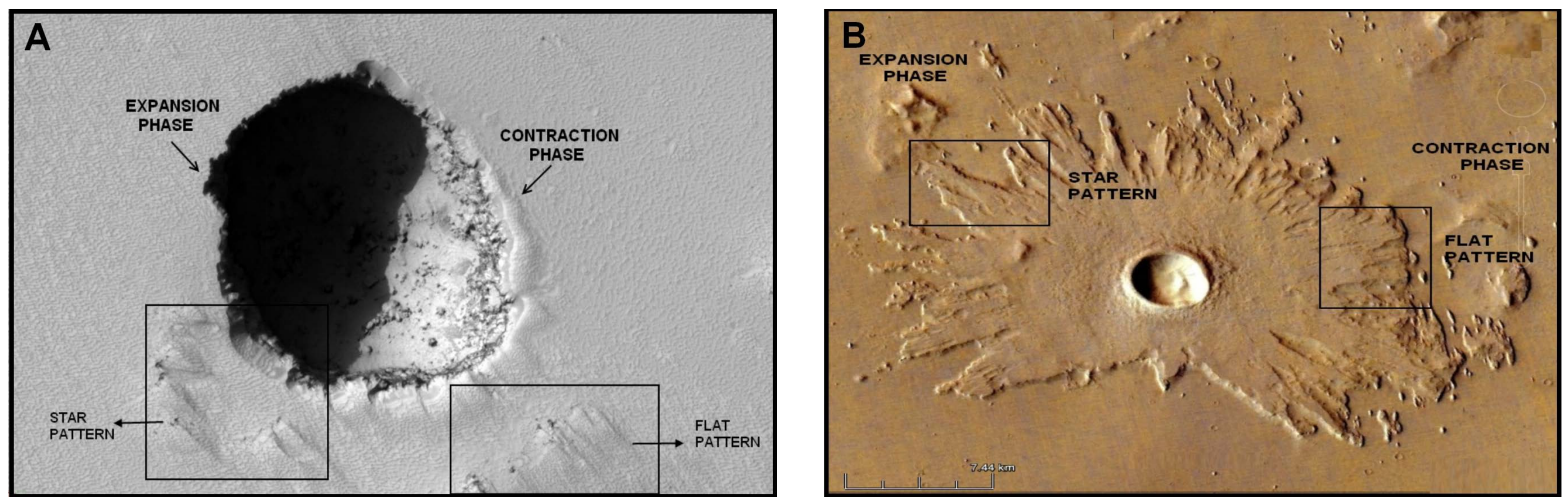

Figure 26. (A), (B) visible dipole energy generate in the interior of MARS impact craters: Due to inward and upward motion of the target rocks. Expansion or centrifugal energy in one semicircle crater area its balanced with contraction centripetal energy in the opposite one, expansion-centrifugal zones show well defined edge rim wall borders with like star pattern dissemination, contraction centripetal zones evidence stratification hyper densities with flattened edges.

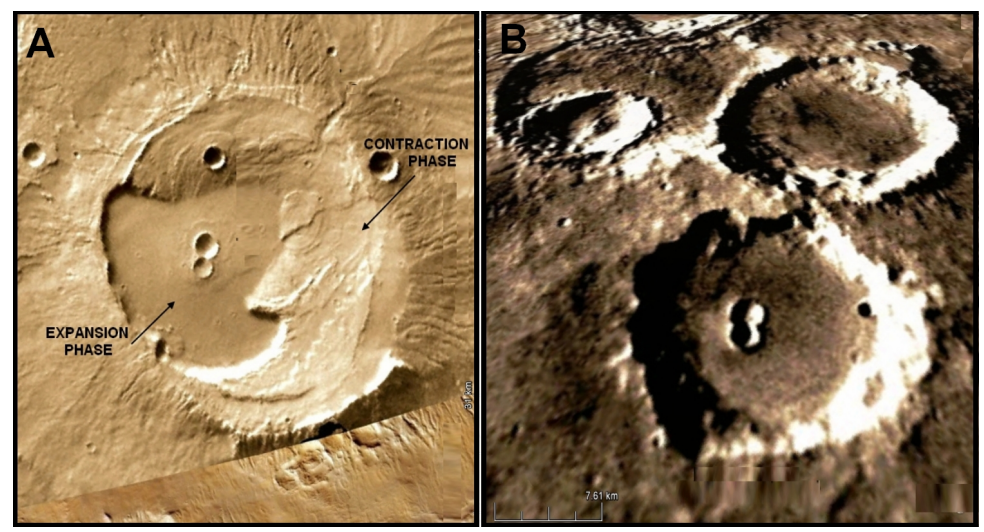

Figure 27. (A), (B) Illustrate dipole energy generate in the interior of MARS impact craters, Expansion-centrifugal zones show well defined edge rim wall borders and contraction centripetal zones with stratification hyper densities with flattened edges.

c) This embryoid body structure originates in the crater contraction collision area by the vibratory action of echo waves in response to a light-electromagnetic field of different frequencies, visible by the presence of 12 parallel black and white intercalated bands positioned in the polar head area (Figure 21).

d) The light-electromagnetic field produces in the polar head area, ectodermal perfect linear white matter cortex, spatial and morphologically related with the white body trunk endodermal matter. The polar tail linear black matter ectodermal area is spatial and morphologically related with the black head brain endodermal matter. Self- 


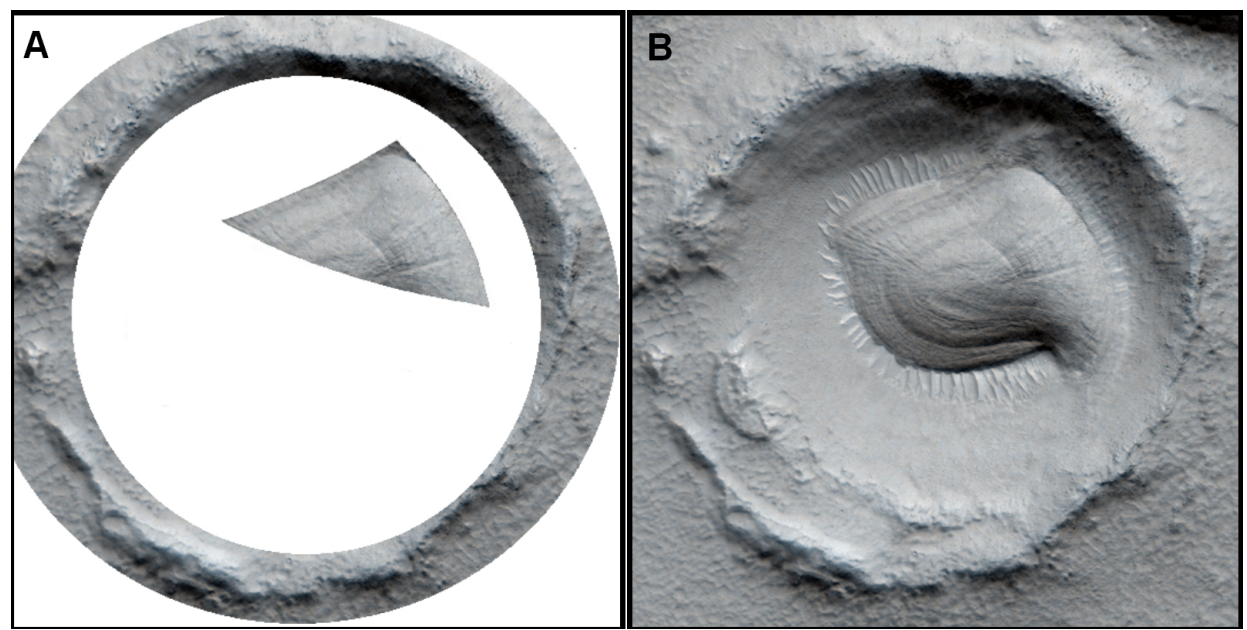

Figure 28. Mars HIRISE image that illustrate impact crater with triangle structure in their interior.

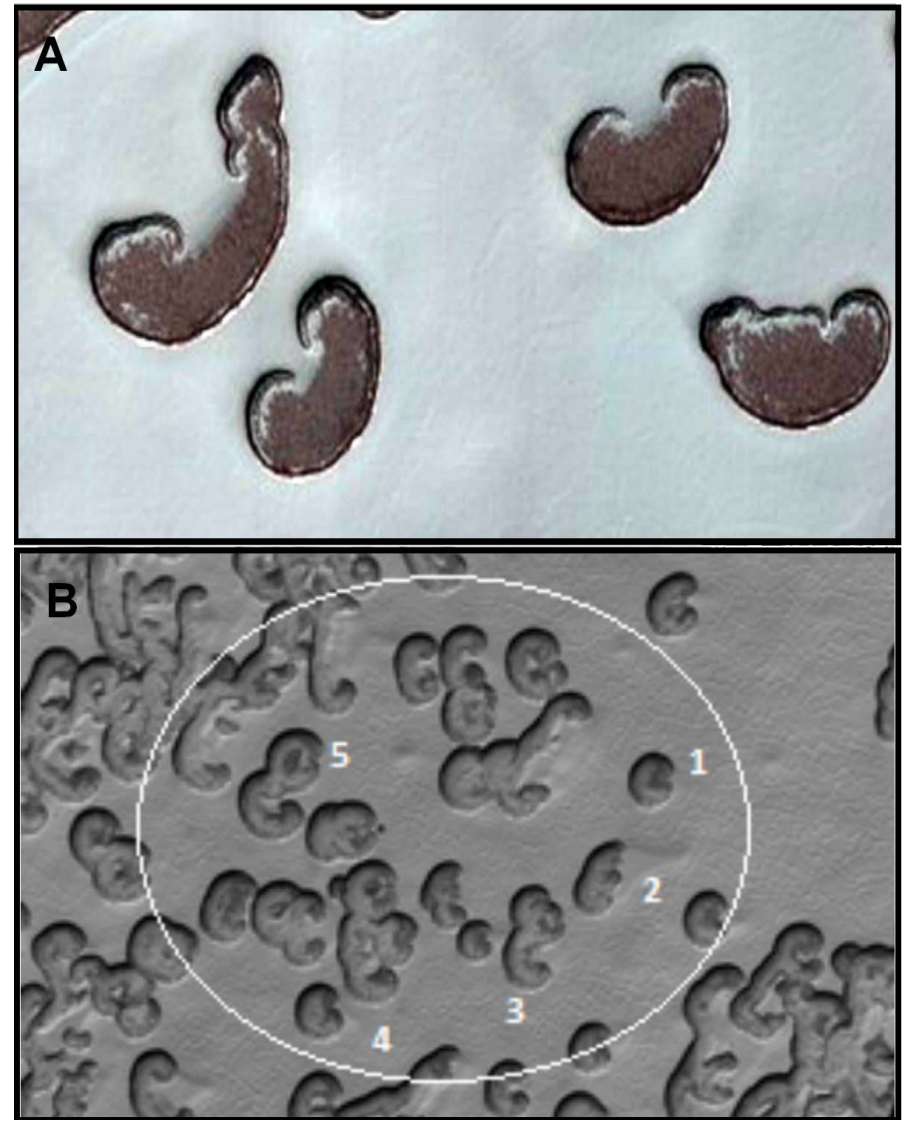

Figure 29. (A), (B) Mars HIRISE infrared image that illustrate perfect sequence of growth and development of collision embryoid bodies in the Mars south pole cap.

assembly pattern that denoting chiral order (Figure 21).

This intricate spatial organization reminds us of the organization of nervous systems similar to today's vertebrates. We are in the presence of a real unique structure with bio signature specialized pattern in the interior of collision impact crater.

Additionally, it is pertinent to emphasize that reviewing the literature, we are the first to document geometric 


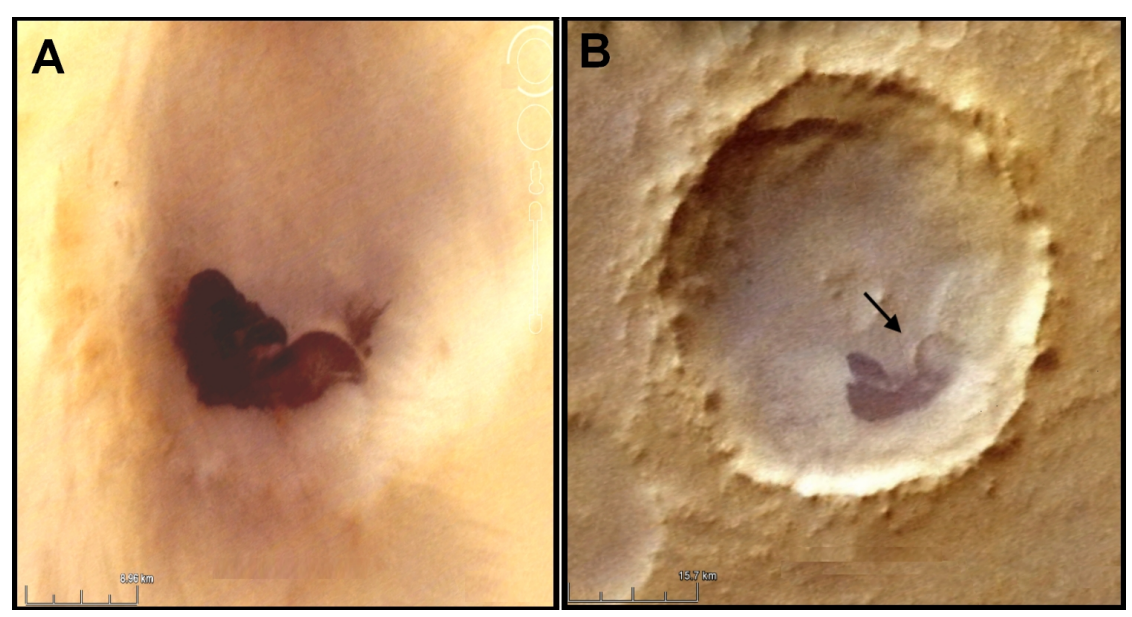

Figure 30. Mars HIRISE image that illustrate impact crater with embryoid structure in their interior.

organization within collision impact craters, collision events generate geometric order, and it is a real, measurable and predictable fact.

For us, GTCHC complexes are the most viable geometric platform of life, as for many biological systems with intricate function; the mesoscale geometry is optimized to provide functionality from basic physical principles, that highlights the importance of patterns and morphology in develop order systems. Order must precede the origin of life. We propose, on the basis of our documented, real, measurable, scalable and predictable GTCHC complexes these sequential events: Collision impact events generate irreducible geometric matrix in the interior of impact craters. In this platform, carbon deposit molecules in rocks are activated by bombardment of plasma and grow [10]. Scientists say that although there is a lot of carbon in the atmosphere, there should be a whole lot more is it trapped in the rocks; carbon hypertrophic geo bands of helicoidal patterns structuring organized layers, the hypertrophy of these helices generated from the wall of impact craters produces morphologic stratifications that we called collision bodies. Carbon nanostructures grow under extreme particle bombardment and represent the primordial dipole organization in which particles and molecules take positional identity: ventral, caudal, and dorsal forms of pre-existence of life, as well as geo molecules in transit to biomolecules.

Additionally, such impacts would have caused massive disruption of the Earth's crust, with extensive fracture zones that were filled with hot circulating water. Such hydrothermal areas could have created complex zones with many exotic minerals [11]. Life originated as a result of natural processes that exploited early Earth's raw materials. Scientific models of life's origins almost always look to minerals for such essential tasks as the synthesis of life's molecular building blocks or the supply of metabolic energy [12]-[17].

Documented images put in clear evidence here, that MOA has morphological characteristics that make it a unique place on Mars, though this nested structures, appear in a medium of inert craters, MOA, evidence in their structures implicit dynamic sequential order inherent to collective memory activation. This visible dynamic sequentially order represent by itself biosignature pattern.

We call on astrophysicists and astrobiologists to analyze possibilities for choosing MOA as a unique recognition place of definitive morphology biosignature to explore life on future Mars expeditions. Impact craters may become a refuge for life, or even a potential birthplace for life's origin. For a habitat within a crater to remain "home sweet home," there must be a constant supply of water and nutrients. A crater etched into the landscape of a planet is not only the fingerprint of the rogue Solar System body that caused the collision but also provides clues to the impact on life after such an event.

\section{Conclusions}

In conclusion, collision-impact craters generate in their interior invariant irreducible geometric matrix of triangular chiral hexagonal structures in interphase with morphology biosignature patterns, related to the development of visible contraction-expansion dipole energy and due to an inward and upward motion of the target rocks. Geometric order could potentially be life-bearing. 
Life probably was created and sustained at the very edge of chaos. In consequence, the earth crater impact zones, far from having generated destruction in the past, could be, based on our criteria, the most plausible nesting place for the origin of life in our planet, where MFs, tons of high-energy particles may flow through space turbulence and create heavenly zones of irreducible geometry order. Crater, therefore, is a step forward in understanding how collisions influence life, both on Earth and on other planets. We were pleased that the correct pattern image algorithm predicted the identification of irreducible geometry matrix of GTCHC complexes in Martian impact craters. Cancer can inform astrobiology. Further interdisciplinary collaboration must be carried out to study these impact craters geometric geo-logical structures, ancient sediments and rocks that could provide insights into antecedents of life.

\section{Acknowledgements}

The author thanks Dr. Juan Carlos Triana and Dr. Jorge Ovidio Cruz, executive directors of the Hospital Department of Villavicencio and Hospital Department of Granada, for their financial and logistic support of this research.

\section{References}

[1] Diaz, J., Jaramillo, N. and Murillo, M. (2007) Geometric Triangular Chiral Hexagon Crystal-Like Complexes Organization in Pathological Tissues Biological Collision Order. PLoS ONE, 2, e1282. http://dx.doi.org/10.1371/journal.pone.0001282

[2] Diaz, J. and Murillo, M. (2009) Framework of Collagen Type I Vasoactive Vessels Structuring Invariant Geometric At-tractor in Cancer Tissues: Insight into Biological Mag-netic fields. PLoS ONE, 4, e4506. http://dx.doi.org/10.1371/journal.pone.0004506

[3] Diaz, J., Murillo, M. and Barrero, A. (2011) Intercellular Cancer Collisions Generate an Ejected Crystal Comet Tail Effect with Fractal Interface Embryoid Body Reassembly Trans-formation. Cancer Management and Research, 3, 143155.

[4] Diaz, J. and Murillo, M. (2012) Phenotype Characterization of Embryoid Body Structures Generated by a Crystal Comet Effect Tail in an Intercellular Cancer Collision Scenario. Cancer Management and Research, 4, 9-21. http://dx.doi.org/10.2147/CMAR.S25810

[5] Diaz, J. (2013) Electromagnetic Field Released in Collision Impact Events Generate in the Matrix Interface Fractal Scalable Invariant Geometric Triangular Chiral Hexagonal Structures. Open Journal of Geology, 3, 187-200. http://dx.doi.org/10.4236/ojg.2013.33022

[6] Robbins, S.J. and Hynek, B.M. (2012) A New Global Database of Mars Impact Craters $\geq 1$ km: 2. Global Crater Properties and Regional Variations of the Simple-to-Complex Transition. Journal of Geophysical Research: Planets, 117, E06001.

[7] Masantis, L. (2005) Morphological, Structural and Lithological Records of Terrestrial Impacts: An Overview. Australian Journal of Earth Sciences, 52, 509-528. http://dx.doi.org/10.1080/08120090500170427

[8] Folco, L., Di Martino, M., El Barkooky, A., D’Orazio, M., et al. (2010) The Kamil Crater in Egypt. Science, 329, 804807. http://dx.doi.org/10.1126/science.1190990

[9] Diaz, J. (2013) Geometric Triangular Chiral Hexagon Complexes and Clonal Embryogenic Body Organization on the Turin Shroud Crucified Man Image: A Predictable Tissue Response to Injury. Natural Science, 5, 1102-1111. http://dx.doi.org/10.4236/ns.2013.51013

[10] Bystrova, K., et al. (2013) Spontaneous Synthesis of Carbon Nanowalls, Nanotubes and Nanotips Using High Flux Density Plasmas. Carbon, 68, 695-707. http://dx.doi.org/10.1016/j.carbon.2013.11.051

[11] A Crater as an Abode for Life. http://phys.org/news/2013-11-crater-abode-life.html

[12] Paleontologist Presents Origin of Life Theory. http://phys.org/news/2013-10-paleontologist-life-theory.html

[13] Kring, D.A. and Abramov, O. (2005) Impact-Generated Hydrothermal Systems: Potential Sites for Pre-biotic Chemistry and Life on Early Earth and Mars. NASA Astrobiology Conference, Boulder, Colorado, 2005.

[14] O. Abramov and D.A. Kring (2005) Impact-Induced Hydrothermal Activity on Early Mars. Journal of Geophysical Research, 110, E12809. http://dx.doi.org/10.1029/2005JE002453

[15] Schwenzer, S.P. and Kring, D.A. (2006) Impact-Generated Hydrothermal Systems Capable of Forming Phyllosilicates on Noachian Mars. Geology, 37, 1091-1094. http://dx.doi.org/10.1130/G30340A.1

[16] Zurcher, L. and Kring, D.A. (2004) Post-Impact Hydrothermal Alteration in the Yaxcopoil-1 hole, Chicxulub Impact 
Structure, Mexico. Meteoritics and Planetary Science, 39, 1199-1221. http://dx.doi.org/10.1111/j.1945-5100.2004.tb01137.x

[17] Abramov, O. and Kring, D.A. (2007) Numerical Modeling of Impact-Induced Hydrothermal Activity at the Chicxulub Crater. Meteoritics and Planetary Science, 42, 93-112. 